\title{
Produzir saúde suscita adoecimento? As contradições do trabalho em hospitais públicos de urgência e emergência
}

\author{
Generating health elicits illness? The contradictions of work \\ performed in emergency care units of public hospitals
}

Iana Vasconcelos Moreira Rosado ${ }^{1}$

Gláucia Helena Araújo Russo ${ }^{2}$

Eulália Maria Chaves Maia ${ }^{1}$

${ }^{1}$ Programa de PósGraduação em Ciências da Saúde, Centro de Ciências da Saúde, Universidade Federal do Rio Grande do Norte. Av. Gal. Gustavo Cordeiro de Farias s/n, Petrópolis. 59010180 Natal RN Brasil. ianavasconcelos@uern.br ${ }^{2}$ Faculdade de Serviço Social, Universidade do Estado do Rio Grande do Norte.
Abstract In general terms, health is influenced and generated by the interaction of biological, social, economic, political and cultural factors. From this standpoint, the scope of this paper was to analyze the relationship between working in emergency care units of public hospitals and the health/sickness of the professionals who work in them. It involved a quantitative and qualitative survey, in which 240 health professionals (doctors, nurses, social workers, psychologists, dentists, nutritionists, audiologists, physiotherapists and occupational therapists) filled out a questionnaire. All the results acknowledged the importance of work to ensure favorable conditions for good health. However, they highlighted its deleterious physical and mental effects on workers, which included stress, lack of a healthy life-style, high blood pressure and musculoskeletal, gastrointestinal and sleep disorders. It is therefore important to tackle this reality in order to enhance the health of professionals and, consequently, the quality of care provided to the user, since illnesses among health workers are strongly linked to the existing health model in society.

Key words Health, Illness, Work, Occupational health, Public hospitals
Resumo A saúde resulta da interação de fatores biológicos, sociais, econômicos, políticos e culturais. Nessa perspectiva, objetivou-se analisar a relação entre o trabalho nos hospitais públicos de urgência e emergência e o processo de saúde-adoecimento dos profissionais que o executam. Tratase de uma pesquisa de natureza quantiqualitativa, na qual foram aplicados questionários com 240 profissionais (médicos, enfermeiros, assistentes sociais, psicólogos, dentistas, nutricionistas, fonoaudiólogos, fisioterapeutas e terapeutas ocupacionais). Os resultados demonstraram que os sujeitos reconhecem a importância do trabalho para garantia de condições favoráveis à saúde. Entretanto, destacam seus efeitos no desgaste físico e psíquico dos trabalhadores, por impulsionar estresse, ausência de hábitos saudáveis, hipertensão arterial, distúrbios do sono, osteomusculares e gastrintestinais. Torna-se premente enfrentar esta realidade, para potencializar a saúde dos profissionais e, por conseguinte, a qualidade da assistência ao usuário, visto que o adoecimento dos trabalhadores de saúde está fortemente relacionado com o modelo de saúde existente na sociedade.

Palavras-chave Saúde, Doença, Trabalho, Saúde do Trabalhador, Hospitais Públicos 


\section{Introdução}

A saúde, em sua acepção ampliada, consiste em um processo dinâmico e multifacetado, o qual deriva de mecanismos biológicos, influenciados pelas condições de vida e ambientais, pela assistência prestada pelos serviços de saúde e pelo estilo de vida dos sujeitos sociais em determinado contexto histórico ${ }^{1}$.

Sob este prisma, o trabalho configura-se como sendo um dos fatores determinantes da situação de saúde dos trabalhadores, podendo contribuir para seu fortalecimento ou sua deterioração ${ }^{2}$. Como categoria fundante do ser social, ao impulsionar a transformação da realidade, indispensável à reprodução da existência humana, o trabalho intermedia e constrói relações, fomenta a autoprodução do indivíduo e impele a construção de formas de sociabilidade erigidas pelos sujeitos sociais ${ }^{3}$.

$\mathrm{Na}$ sociedade capitalista em particular, o trabalho representa um veículo de exploração e desigualdade social e, por conseguinte, atua muitas vezes como desencadeador ou intensificador de desgaste da saúde ${ }^{4}$. Nesse sentido, encontra-se onipresente na totalidade da vida social, definindo a posição ocupada pelo trabalhador em uma estrutura social hierárquica, com distintas possibilidades de consumo, prazer, saúde-adoecimento e morte ${ }^{5}$.

É relevante salientar que a saúde e a doença não são processos restritos ao sujeito que trabalha, embora o trabalho seja um dos seus determinantes. Logo, os trabalhadores vivenciam formas de adoecer e morrer definidas pelo estilo de vida, sexo, idade, características genéticas e condições ambientais, as quais sobrevêm na população em geral, ao mesmo tempo em que estão propensos a acidentes e doenças cujas incidências decorrem de fatores intrínsecos ao trabalho efetuado ${ }^{6}$.

No caso do adoecimento dos profissionais de saúde relacionado ao trabalho, estes vivenciam os efeitos nefastos à saúde atinentes à forma de estruturação das relações sociais capitalistas, bem como se deparam com situações particulares à sua atividade laborativa. Na área da saúde, a efetivação do trabalho não depende exclusivamente da competência e compromisso do trabalhador; mas também das condições de trabalho, da interação com o usuário e da adesão, acesso e reação deste aos procedimentos e orientações ${ }^{7}$.

Importa salientar que, na realidade brasileira, os profissionais de hospitais públicos de urgência e emergência, de modo geral, lidam com precárias condições de trabalho, como insuficiência de profissionais, materiais, equipamentos, estruturas físicas inadequadas, falta de leitos hospitalares, superlotação e defasagem salarial ${ }^{8,9}$.

Sem desconsiderar os avanços conquistados a partir da implantação do Sistema Único de Saúde (SUS), especialmente em termos de ampliação do acesso aos serviços e consequente melhoria no perfil epidemiológico, persistem diversas fragilidades em sua operacionalização ${ }^{10}$.

Tal situação impõe limitações exógenas à vontade e competência dos profissionais e, ao mesmo tempo, indispensáveis à consecução da sua capacidade laborativa, sujeitando-os ao estresse e sofrimento no trabalho, ao atuar em meio a condições adversas à materialização de uma assistência capaz de atender, com a devida efetividade e qualidade, às demandas da população usuária ${ }^{2,11}$.

Estes fatores, somados aos riscos de acidentes $^{12,13} \mathrm{e}$ às situações inerentes ao âmbito hospitalar - a exemplo do expediente noturno, trabalho em turnos, prolongadas jornadas, excesso e complexidade das tarefas - impulsionam o desgaste físico e emocional dos trabalhadores ${ }^{9,14}$.

Nesse contexto, diversos estudos têm se debruçado sobre os processos de adoecimento relacionados ao trabalho em saúde. Grande parte destes particulariza a análise sobre determinada profissão, sobretudo os trabalhadores de enfermagem, sendo priorizado o enfoque quantitativo. A abordagem quantitativa é imprescindível ao conhecimento do perfil epidemiológico de morbidade e mortalidade associada ao trabalho; entretanto, é relevante agregar a estas informações a apreensão dos sentidos atribuídos pelos próprios trabalhadores à relação entre o trabalho que executam e sua situação de saúde-adoecimento, tal como nos propomos a efetivar neste artigo.

Além disso, as análises aqui empreendidas evidenciam os discursos dos sujeitos pertencentes a diversas categorias profissionais envolvidas no trabalho coletivo em saúde, acrescentando importantes contribuições à produção científica. Sua significância se concentra, ainda, no fato de desvelar a realidade particular dos hospitais públicos de urgência e emergência no Rio Grande do Norte, em um contexto de profundas limitações na qualidade e efetividade da assistência à saúde, tendo sido decretado, no ano de 2012, estado de calamidade pública nessa área, conforme documento expedido pelo governo estadual ${ }^{15}$.

Diante do exposto, objetiva-se analisar a relação entre o exercício profissional nos hospitais públicos de urgência e emergência e as situações de saúde-adoecimento dos sujeitos que o execu- 
tam, considerando as múltiplas determinações desse processo.

\section{Metodologia}

Esta pesquisa consiste em um estudo quantiqualitativo efetivado no Hospital Monsenhor Walfredo Gurgel (HMWG) e no Hospital Regional Tarcísio Maia (HRTM), situados em Natal e Mossoró, respectivamente, no Estado do Rio Grande do Norte, Nordeste brasileiro. Tais instituições integram a rede hospitalar estadual de urgência e emergência, composta de sete hospitais, dos quais foram delimitados os que absorvem maior quantidade de profissionais e de atendimentos.

A opção metodológica de recorrer a dados quantitativos e qualitativos assenta-se no entendimento de que o desvelamento dos determinantes da saúde-adoecimento dos profissionais não prescinde da mensuração quantitativa do perfil sociodemográfico e das condições de trabalho destes. Ao mesmo tempo, estes elementos são insuficientes para captar a multiplicidade de aspectos subjetivos que interagem neste processo. Isto posto, o fenômeno estudado incorpora processos históricos, culturais, políticos e ideológicos incapazes de se desvelarem completamente, em sua dinamicidade, complexidade e multidimensionalidade, em um dado numérico ou cálculo estatístico $^{16}$.

Daí a necessidade de atribuir ênfase à análise qualitativa, visando adentrar o mundo dos significados, motivações, valores e atitudes que mobilizam ações e interações humanas em suas particularidades e interseções com a totalidade social. Logo, esta análise fundamenta-se na compreensão do quantitativo e do qualitativo como complementares e essenciais no percurso da sucessiva e inacabada aproximação do conhecimento acerca da dinâmica e contraditória realidade social ${ }^{16,17}$.

Assim, do ponto de vista quantitativo, a população alvo do estudo constituiu-se de 861 profissionais. Diante desse universo, a amostra foi definida de forma estratificada proporcional, aglutinando 240 trabalhadores, sendo 155 inseridos no HMWG e 52 atuantes no HRTM. Os participantes foram abordados nas próprias instituições, entre novembro de 2012 a julho de 2013, por meio da busca espontânea, contemplando os vários turnos e dias da semana.

Foram delimitados como critérios de inclusão: a) atuação nas instituições mediante vínculos de trabalho formal; b) exercício de profissões de nível superior com contato direto com usuários, especificamente: Medicina, Enfermagem, Serviço Social, Odontologia, Psicologia, Fisioterapia, Fonoaudiologia, Nutrição e Terapia Ocupacional.

Na pesquisa de campo aplicou-se questionário elaborado pelas pesquisadoras, composto de perguntas fechadas e abertas. A parte objetiva contemplou informações sociodemográficas e profissionais, além de uma lista de fatores intrínsecos ao trabalho que possivelmente influenciariam (favorável ou desfavoravelmente) a saúde dos participantes. Utilizou-se a estatística descritiva para a mensuração dos dados que emergiram destas questões.

Em relação às perguntas abertas, tendo em vista sua pertinência com as análises contidas neste artigo, delimitaram-se as seguintes: (i). Existe relação entre o seu trabalho e suas condições de saúde? Se sim, de que forma ou que tipo de relação? (ii). De alguma forma o seu trabalho dificulta sua vida social/familiar? Por quê?

Os conteúdos que emergiram de tais questões foram interpretados qualitativamente, mediante análise de conteúdo, conforme proposto por Bar$\operatorname{din}^{18}$. Na fase de pré-análise foi realizada leitura flutuante, como forma de aproximação preliminar com os dados. Assim, por meio de atentas e reiteradas leituras, foi possível apropriar-se de sua riqueza e complexidade. Em seguida, realizou-se procedimentos de sistematização, codificação e categorização, em busca de identificar semelhanças e dissonâncias expressas nos discursos, sendo selecionadas as respostas mais significativas. Finalizando este processo, efetivou-se a interpretação dos resultados fundamentada na revisão da literatura sobre o tema.

$\mathrm{Na}$ apreensão e interpretação dos dados, considerou-se não apenas sua frequência, mas prioritariamente as subjetividades neles presentes, posto que a análise qualitativa se debruça sobre os significados dos discursos, o que nem sempre se explicita pela sua repetição.

Importa ressaltar que os sujeitos apresentam heterogeneidades sociais, econômicas, culturais e profissionais. Encontram-se imersos, na condição de construtos e construtores de um trabalho coletivo que os aproxima, mas que pode também distanciá-los. Por subsecutivo, apresentam singularidades em suas vivências no trabalho e fora dele, assim como nas formas de concebê-las e significá-las.

Tal fato, somado ao elevado número de participantes, nos demandou grande esforço de análise para compreender, interpretar e sistematizar os resultados, evitando menosprezar semelhan- 
ças, diferenciações e divergências de perspectivas expressas pelos profissionais. Sob este prisma, conforme enfatiza Minayo ${ }^{19}$, um relato coletivo não apresenta homogeneidade, mas abrange e visibiliza interesses e visões envolvidas, atribuindolhes lugar e expressão.

A investigação foi efetivada em consonância com os princípios éticos de pesquisa com seres humanos, conforme as indicações da Declaração de Helsinque e das Resoluções do Conselho Nacional de Saúde no 196/1996 20 e no 251/1997²1. O estudo foi aprovado pelo Comitê de Ética em Pesquisa da Universidade do Estado do Rio Grande do Norte. Os participantes foram esclarecidos acerca dos objetivos e procedimentos da pesquisa e, após manifestação de aceite, responderam os questionários, mediante assinatura do Termo de Consentimento Livre e Esclarecido.

\section{Resultados e Discussão}

\section{Caracterização dos sujeitos que vivenciam e atribuem significado aos processos de saúde-adoecimento relacionados ao trabalho}

Os sujeitos integram diferentes profissões envolvidas no trabalho coletivo em saúde, conforme demonstra a Tabela 1. Apresentam faixa etária diversificada, registrando-se média de 44,5 anos (desvio padrão $\pm 11,24$ ). A maioria vivencia relação conjugal $(63,4 \%)$ e possui filhos $(67,1 \%)$.

Predominam $(60,4 \%)$ mulheres na totalidade da amostra, fato que se registra na maioria das profissões abrangidas neste estudo, exceto Medicina e Odontologia (cirurgia bucomaxilofacial), nas quais $66,4 \%$ e $100 \%$ dos participantes, respectivamente, são homens. A preponderância de mulheres na amostra coaduna com registros da feminização da força de trabalho envolvida na assistência à saúde, amplamente explicitada na literatura ${ }^{11,22-24}$.

Registrou-se, ainda, que 95,4\% dos participantes possuem vínculo empregatício efetivo, enquanto a remuneração pelo trabalho nos hospitais varia entre $R \$ 1.200,00$ e $R \$ 16.000,00$, contabilizando-se uma média salarial de $\mathrm{R} \$ 5.369,34$ (desvio padrão $\pm \mathrm{R} \$ 2.500,93$ ). Apesar da elevada incidência de não resposta neste item $(30,8 \%)$, os resultados sinalizam expressiva diferenciação salarial. Identificou-se, também, heterogeneidade na renda familiar, estando as maiores concentrações nas faixas de 6 a $10(27,1 \%)$ e acima de 25 salários mínimos $(23,8 \%)$.
Acerca da frequência do usufruto de férias, considerando o afastamento simultâneo de todas as atividades, constatou-se que $25 \%$ dos profissionais nunca ou apenas raramente o fazem. Por outro lado, uma quantidade significativa $(78,5 \%)$ trabalha de forma frequente em finais de semana e feriados e participa $(53,4 \%)$ de plantões noturnos.

Concernente à experiência de trabalho, grande parte dos profissionais atua há bastante tempo na saúde, bem como nos hospitais estudados, o que os qualifica a analisar o trabalho nestes espaços e os impactos deste na sua saúde.

\section{Interfaces entre trabalho e processos de saúde-adoecimento dos profissionais: no limiar da proteção e do desgaste}

Os profissionais, em sua maioria, vislumbram haver relação entre o trabalho que desenvolvem e sua situação de saúde, muito embora esta associação seja vista de formas distintas. Vale assinalar que $13,8 \%$ dos sujeitos não se manifestou sobre esta questão, o que pode ser explicado pela indisponibilidade de tempo para preenchimento dos questionários, especialmente por se tratar de questão aberta.

De acordo com os dados exibidos na Tabela 2, destacaram-se posicionamentos acerca dos impactos negativos do trabalho na saúde, não obstante alguns profissionais o tenham enaltecido como potencializador da qualidade de vida, enquanto outros explicitaram influências favoráveis e desfavoráveis na sua situação de saúde.

Esta quantificação dos dados esboça um panorama da percepção dos sujeitos sobre a relação entre o trabalho que exercem e sua condição de saúde-adoecimento, possibilitando uma preliminar aproximação com o fenômeno observado. Compreensão mais aprofundada acerca dos processos vivenciados pelos profissionais e da significação atribuída a estes, será explanada mediante análise dos depoimentos destes sujeitos.

Desse modo, observou-se que prevaleceu o entendimento do trabalho como fonte de desgaste da saúde, em função de atributos inerentes à própria atividade profissional e ao ambiente em que ela se realiza. Dentre os aspectos intrínsecos às funções desempenhadas, destacaram-se como potenciais impulsionadores de problemas osteomusculares: esforço físico, procedimentos repetitivos e extenuante permanência em pé. Fatores especialmente relevantes se considerados os registros emitidos pelos profissionais acerca das condições não ergonômicas nos serviços investigados.

Pela condição de trabalho adquiri dores lombares crônicas e déficit de audição (dentista, 
Tabela 1. Perfil ocupacional dos profissionais de hospitais públicos de urgência e emergência do Estado do Rio Grande do Norte, Nordeste brasileiro, 2013.

\begin{tabular}{|c|c|c|c|c|c|c|}
\hline \multirow[b]{2}{*}{ Variáveis } & \multicolumn{2}{|c|}{ HRTM } & \multicolumn{2}{|c|}{ HMWG } & \multicolumn{2}{|c|}{ Total } \\
\hline & $\mathbf{n}$ & $\%$ & $\mathbf{n}$ & $\%$ & n & $\%$ \\
\hline \multicolumn{7}{|l|}{ Profissão } \\
\hline Médico & 22 & 30,6 & 85 & 50,6 & 107 & 44,6 \\
\hline Enfermeiro & 22 & 30,6 & 36 & 21,4 & 58 & 24,2 \\
\hline Assistente Social & 11 & 15,3 & 11 & 3,6 & 22 & 9,2 \\
\hline Fisioterapeuta & 5 & 6,9 & 11 & 6,5 & 16 & 6,7 \\
\hline Nutricionista & 2 & 2,8 & 10 & 6,0 & 12 & 5,0 \\
\hline Cirurgião-dentista & 4 & 5,6 & 6 & 3,6 & 10 & 4,2 \\
\hline Fonoaudiólogo & 1 & 1,4 & 5 & 3,0 & 6 & 2,5 \\
\hline Psicólogo & 2 & 2,8 & 3 & 1,8 & 5 & 2,1 \\
\hline Terapeuta Ocupacional & 3 & 4,2 & 1 & 6 & 4 & 1,7 \\
\hline \multicolumn{7}{|l|}{ Nível de formação } \\
\hline Graduação & 20 & 28,6 & 37 & 22,0 & 57 & 23,9 \\
\hline Especialização/residência & 47 & 67,1 & 120 & 71,4 & 167 & 70,2 \\
\hline Mestrado/Doutorado & 3 & 4,3 & 11 & 6,6 & 14 & 5,8 \\
\hline \multicolumn{7}{|l|}{ Tempo de trabalho na saúde } \\
\hline \multicolumn{7}{|l|}{ Inferior a 1 ano } \\
\hline 1 a 2 anos & 2 & 2,8 & 2 & 1,2 & 4 & 1,7 \\
\hline 3 a 5 anos & 9 & 12,5 & 24 & 14,3 & 33 & 13,8 \\
\hline 6 a 10 anos & 10 & 13,9 & 25 & 14,9 & 35 & 14,6 \\
\hline Acima de 10 anos & 51 & 70,9 & 117 & 69,7 & 168 & 70 \\
\hline \multicolumn{7}{|l|}{ Tempo de trabalho na instituição } \\
\hline Inferior a 1 ano & 3 & 4,2 & 18 & 10,8 & 21 & 8,8 \\
\hline 1 a 2 anos & 13 & 18,1 & 9 & 5,4 & 22 & 9,2 \\
\hline 3 a 5 anos & 17 & 23,6 & 50 & 29,9 & 67 & 28.0 \\
\hline 6 a 10 anos & 6 & 8,3 & 12 & 7,2 & 18 & 7,5 \\
\hline Acima de 10 anos & 33 & 45,8 & 78 & 46,7 & 111 & 46,4 \\
\hline \multicolumn{7}{|c|}{ Jornada de trabalho semanal na instituição } \\
\hline $40 \mathrm{~h}$ & 43 & 60,6 & 106 & 66,3 & 149 & 64,5 \\
\hline $30 \mathrm{~h}$ & 24 & 33,8 & 27 & 16,9 & 51 & 22,1 \\
\hline $20 \mathrm{~h}$ & & & 10 & 6,3 & 10 & 4,3 \\
\hline Outra & 4 & 5,6 & 17 & 10,6 & 21 & 9,1 \\
\hline \multicolumn{7}{|c|}{ Total de horas dedicadas ao trabalho semanalmente } \\
\hline Até 40 horas & 19 & 8,1 & 53 & 22,5 & 72 & 30,6 \\
\hline$>40$ até 60 horas & 26 & 11,0 & 61 & 25,8 & 87 & 36,9 \\
\hline$>60$ até 80 horas & 18 & 7,6 & 29 & 12,3 & 47 & 19,9 \\
\hline$>80$ até 100 horas & 5 & 2,1 & 10 & 4,2 & 15 & 6,4 \\
\hline$>100$ horas & 3 & 1,3 & 8 & 3,4 & 11 & 4,7 \\
\hline
\end{tabular}

HMWG). O processo de trabalho tem se refletido em dores musculares, coluna e cefaleias que não sentia antes daqui (fisioterapeuta, HRTM). [...] ficar muito tempo em pé provoca dor nos membros inferiores, articulações etc. (enfermeira, HMWG).

Os distúrbios osteomusculares vêm se apresentando, no âmbito nacional e internacional, como preocupante problema relacionado ao trabalho em saúde. Apesar de sua etiologia multifatorial, têm demonstrado associação com situações que perpassam o trabalho em várias profissões de saúde, marcadas pela mobilização excessiva do sistema musculoesquelético, devido ao empenho constante de esforço físico, movimentos repetitivos e posições inadequadas ${ }^{24,25}$.

Foram apontados, também, riscos atinentes ao espaço hospitalar, tais como ambiente insalubre, turnos prolongados e expedientes noturnos.

Devido à exposição a doenças infectocontagiosas tenho muitas sinusites e também, por passar muito tempo em pé, dores na coluna (enfermeira, HMWG). [...] Gera estresse, ansiedade, fiquei hi- 
pertensa e tinha enxaqueca após plantão noturno. Fiquei obesa de tanto comer à noite nos plantões e ao longo da vida comer 'correndo' de um plantão para outro (médica, HMWG).

A literatura registra várias manifestações do caráter nefasto do trabalho para a saúde, incluindo-se a influência deste no desencadeamento da hipertensão arterial. Ainda que este problema resulte da interação entre mecanismos genéticos, biológicos, comportamentais e ambientais, lidar continuamente com condições de trabalho estressantes pode impulsionar episódios de elevação dos níveis pressóricos e contribuir para a instalação do quadro hipertensivo. Essa associação é evidenciada em estudo realizado com pro- fissionais de serviços de atendimento pré-hospitalar no Sudeste brasileiro, no qual se constatou que trabalhar frequentemente cansado elevou a chance de os sujeitos apresentarem pressão arterial alterada ${ }^{14}$.

No que concerne ao trabalho noturno, este foi bastante explicitado pelos sujeitos como fator de desgaste da saúde, principalmente por acarretar distúrbios do sono; mas, também, irritabilidade, cefaleia, cansaço, obesidade, entre outros. Eis alguns relatos: [...] tenho dificuldade para dormir, devido à questão dos noturnos (enfermeira, HMWG). Após aderir à escala noturna, aumentou minha irritabilidade e as crises de gastrite (enfermeira, HMWG).

Tabela 2. Relação entre trabalho e saúde, segundo profissionais de hospitais públicos de urgência e emergência do Estado do Rio Grande do Norte, Nordeste brasileiro, 2013.

\begin{tabular}{|c|c|c|c|c|c|c|c|c|c|}
\hline \multirow[b]{2}{*}{ Profissão } & \multicolumn{8}{|c|}{ Relação saúde/adoecimento no trabalho } & \multirow[b]{2}{*}{ Total } \\
\hline & & $\begin{array}{c}\text { O trabalho } \\
\text { fortalece a } \\
\text { saúde }\end{array}$ & $\begin{array}{c}\text { O trabalho } \\
\text { debilita a } \\
\text { saúde }\end{array}$ & $\begin{array}{c}\text { O trabalho } \\
\text { fortalece e } \\
\text { debilita a } \\
\text { saúde }\end{array}$ & $\begin{array}{l}\text { O trabalho } \\
\text { é afetado } \\
\text { pela saúde/ } \\
\text { doença }\end{array}$ & $\begin{array}{l}\text { Não há } \\
\text { relação }\end{array}$ & $\begin{array}{c}\text { Sem } \\
\text { apreciação } \\
\text { definida }\end{array}$ & $\begin{array}{c}\text { Sem } \\
\text { resposta }\end{array}$ & \\
\hline \multicolumn{10}{|l|}{ Médico } \\
\hline & $\mathrm{n}$ & 10 & 53 & 01 & 03 & 19 & 06 & 15 & 107 \\
\hline & $\%$ & 4,1 & 22,1 & 0,4 & 1,3 & 7,9 & 2,5 & 6,3 & 44,6 \\
\hline \multicolumn{10}{|l|}{ Enfermeiro } \\
\hline & $\mathrm{n}$ & 03 & 29 & 01 & & 12 & 04 & 09 & 58 \\
\hline & $\%$ & 1,3 & 12,1 & 0,4 & & 5,0 & 1,6 & 3,8 & 24,2 \\
\hline \multicolumn{10}{|l|}{ Assistente social } \\
\hline & $\mathrm{n}$ & & 14 & & & 02 & 03 & 03 & 22 \\
\hline & $\%$ & & 5,8 & & & 0,8 & 1,3 & 1,3 & 9,2 \\
\hline \multicolumn{10}{|c|}{ Cirurgião-dentista } \\
\hline & $\mathrm{n}$ & 01 & 07 & & & 02 & & & 10 \\
\hline & $\%$ & 0,4 & 2,9 & & & 0,8 & & & 4,1 \\
\hline \multicolumn{10}{|l|}{ Psicólogo } \\
\hline & $\mathrm{n}$ & 01 & 03 & & & & 01 & & 05 \\
\hline & $\%$ & 0,4 & 1,3 & & & & 0,4 & & 2,1 \\
\hline \multicolumn{10}{|l|}{ Fonoaudiólogo } \\
\hline & $\mathrm{n}$ & & 03 & & & 01 & 01 & 01 & 06 \\
\hline & $\%$ & & 1,3 & & & 0,4 & 0,4 & 0,4 & 2,5 \\
\hline \multicolumn{10}{|l|}{ Fisioterapeuta } \\
\hline & $\mathrm{n}$ & & 13 & & & & 01 & 02 & 16 \\
\hline & $\%$ & & 5,4 & & & & 0,4 & 0,8 & 6,6 \\
\hline \multicolumn{10}{|c|}{ Terapeuta ocupacional } \\
\hline & $\mathrm{n}$ & & 02 & 01 & & & & 01 & 04 \\
\hline & $\%$ & & 0,8 & 0,4 & & & & 0,4 & 1,6 \\
\hline \multicolumn{10}{|l|}{ Nutricionista } \\
\hline & $\mathrm{n}$ & & 08 & 01 & & & 02 & 01 & 12 \\
\hline & $\%$ & & 3,3 & 0,4 & & & 0,8 & 0,4 & 5,0 \\
\hline \multicolumn{10}{|l|}{ Total } \\
\hline & $\mathrm{n}$ & 15 & 132 & 04 & 03 & 36 & 18 & 32 & 240 \\
\hline & $\%$ & 6,3 & 55,0 & 1,6 & 1,3 & 15,0 & 7,5 & 13,3 & 100 \\
\hline
\end{tabular}


Este resultado condiz com estudos ${ }^{26,27}$ que relatam acentuação do desgaste gerado pelo trabalho quando desenvolvido durante a noite, em decorrência da ruptura do ciclo circadiano, das dificuldades em usufruir do descanso diurno após plantão, dentre outros problemas. Ressaltase que os efeitos da exposição prolongada a esta vivência laboral podem se manifestar tardiamente. A literatura recente registra indícios de que o exercício reiterado do trabalho em turnos noturnos pode estar associado ao risco de ser acometido por doenças cardiovasculares ${ }^{28,29}$, assim como por alguns tipos de câncer ${ }^{30,31}$.

É notório que os serviços de urgência e emergência não prescindem do expediente noturno; contudo, este horário de trabalho demanda estratégias institucionais e pessoais que minimizem o desgaste ocasionado por ele, tais como: reorganização da rotina, ambientes adequados, remuneração diferenciada e iniciativas propiciadoras da qualidade de vida dos trabalhadores ${ }^{32}$.

A menor concentração de atividades durante a noite condiz com a necessidade de minimização do desgaste para plantonistas noturnos. Porém, é premente também evitar o acúmulo extenuante de tarefas na rotina diurna e o desgaste dele subsequente. Esta reflexão se fundamenta em pesquisa, cujos resultados demonstraram que níveis mais elevados de estresse e pior qualidade do sono prevaleceram entre os profissionais diurnos, fato relacionado com a concentração das atividades sob sua responsabilidade neste horário ${ }^{33}$.

Não obstante, a sobrecarga de trabalho apareceu nos relatos das diversas categorias, com queixas de cansaço e indisponibilidade de tempo para descanso, lazer, atividades físicas, práticas alimentares saudáveis, convívio social e familiar, além de outros cuidados com a própria saúde.

Muitas vezes sou impedida de realizar atividades que fazem bem à saúde devido ao tempo que preciso estar no trabalho, como, por exemplo, mais atividade fisica, aula de violão, etc. (assistente social, HMWG). A sobrecarga de trabalho interfere de forma significativa nas condições de saúde [especialmente psíquica] e em minha qualidade de vida (psicóloga, HMWG). Muito trabalho diminui a minha qualidade de vida (nutricionista, HMWG).

Os relatos sobre extenuantes cargas de trabalho denotam a importância da manutenção de trabalhadores em número suficiente para atender às demandas institucionais, bem como de remunerações condizentes com suas necessidades e qualificação, o que poderia minimizar o acúmulo de atividades e vínculos profissionais. Destarte, a sobrecarga resulta das configurações do trabalho nos hospitais investigados, mas as transcende, pois é influenciada pela forma de organização e significação do trabalho (e seu equivalente monetário) na sociabilidade em que vivemos. Denota, também, cumulativamente, as vivências em diferentes espaços profissionais ${ }^{34,35}$.

O elevado nível de estresse no trabalho também foi amplamente destacado como um dos grandes vilões no que se refere à saúde: Já enfartei por stress (médico, HMWG). Às vezes, a sobrecarga e o estresse me influenciam com resfriados, dores gástricas etc. (nutricionista, HMWG).

O estresse ocupacional deprecia a qualidade de vida e, por conseguinte, a situação de saúde dos trabalhadores, sendo reconhecida sua significância, por exemplo, no desencadeamento da insônia. Esta correlação apresentou-se significativa em estudo com enfermeiros de hospital universitário no Sudeste brasileiro, no qual níveis mais elevados de estresse estiveram associados a piores qualidades do sono ${ }^{33}$.

Além dos aspectos mencionados, foram explicitados fatores de desgaste relacionados ao ambiente institucional e que são passíveis de alteração, a depender da forma de gestão da política de saúde. São estes: precárias condições de trabalho, inexistência de planejamento ergonômico, relações autoritárias, inadequações na estrutura física, baixos salários, entre outros. Sob este prisma, sobressaem-se relatos acerca das condições de trabalho adversas e, principalmente, das suas consequências para a saúde de profissionais e usuários.

No meu trabalho estou exposta a muitos riscos ocupacionais [biológicos, químicos, ergonômicos etc.], como também fico com o lado emocional fragilizado pelas injustiças, negligências, desrespeito com os pacientes, bem como com as relações entre os profissionais desarmônicas (enfermeira, HRTM). As grandes limitações e condições precárias de trabalho nos determina ansiedade, tristeza, sentimento de impotência (médica, HMWG). [...] desde dores na coluna, geradas pela atividade, até o mal-estar psíquico com as más condições de trabatho e remuneração não condizente (fisioterapeuta, HMWG).

Percebe-se, assim, que lidar com estas adversidades, especialmente em serviços de urgência e emergência, gera conflitos pessoais, dilemas éticos e sofrimento psíquico nos profissionais, ao protagonizarem uma assistência à saúde imersa em incongruências entre os procedimentos necessários para recuperar a saúde dos usuários e os entraves institucionais impeditivos de sua plena efetivação². 
Concernente ao desgaste psíquico associado ao trabalho, estudos apontam os profissionais de saúde entre os trabalhadores propensos a desenvolverem síndrome de burnout, manifestada pela junção de exaustão emocional, despersonalização e baixa realização pessoal. Apesar de multifatorial, esta síndrome mantém estreita relação com níveis elevados de tensão e desgaste no trabalho, impulsionados por fatores como sobrecarga, estresse e dissonância entre esforço desprendido e seus resultados e recompensas ${ }^{23,36}$. Observe-se que tais aspectos perpassam o cotidiano de trabalho dos participantes desta pesquisa, o que sinaliza a importância de se atentar também para o risco destes profissionais serem acometidos por burnout.

Quanto ao desgaste físico associado ao trabalho, foram relatados problemas cardiovasculares; osteomusculares; gastrointestinais; respiratórios; distúrbios do sono; amigdalite; redução da capacidade auditiva; cefaleia; cansaço e mal-estar psíquico. Segundo os profissionais, o trabalho está relacionado ao desgaste físico e emocional vivenciado por eles, ocasionando falta de tempo para cuidar da sua própria saúde, especialmente no que tange à adoção de hábitos saudáveis, como praticar atividades físicas, consumir alimentação adequada e desfrutar de lazer.

Resultados similares foram encontrados em estudo qualitativo enfocando os impactos do trabalho na saúde de médicos da Estratégia Saúde da Família no Nordeste brasileiro, os quais aludem comprometimento na dimensão mental e física $^{23}$.

Numa outra perspectiva, a positividade do trabalho foi destacada por alguns profissionais, os quais evidenciaram aspectos objetivos e subjetivos: [...] o trabalho proporciona qualidade de vida - viagem, casa, carro etc., saúde e bem-estar (médica, HMWG). Atualmente, minha relação com o trabalho é favorável, pois me dá satisfação pessoal não afetando minha saúde (psicóloga, HRTM).

Conforme explicitado, o trabalho possui uma dimensão objetiva expressa na recompensa financeira, propiciadora de bens entendidos como necessários a uma vida saudável, assim como uma dimensão subjetiva, referente à satisfação gerada pela atividade profissional, a qual favorece a autoestima e o equilíbrio emocional e define a identidade e o reconhecimento social.

Vale salientar que o reconhecimento, aliado ao valor simbólico contido na sensação de ser útil ao contribuir para reverter o adoecimento dos usuários, potencializa a saúde dos profissionais ao gerar prazer, mesmo diante das adversida- $\operatorname{des}^{2,37}$. Por outro lado, em nossa sociedade calcada na mercantilização da vida humana, a dimensão material aparece como a mais importante, ao possibilitar a sobrevivência e permitir a aquisição de bens materiais que transformam o ser humano em consumidor e, deste modo, necessário e importante para a sociedade ${ }^{38}$. Isto tende a impelir ao acúmulo de vínculos empregatícios e ao excesso de horas dedicadas ao trabalho, o que pode acabar subsumindo o prazer dele decorrente.

Os discursos apontam para o caráter contraditório do trabalho, haja vista ser imprescindível e prazeroso, ao mesmo tempo em que se torna desgastante e influencia no adoecimento daqueles que o executam. Apesar de ser cansativo, existem momentos bons e a sensação do dever cumprido (nutricionista, HMWG). Exerço minhas atividades com prazer, no entanto, por ser estressante e desgastante, ao longo de quase 3 décadas desenvolvi doenças relacionadas ao stress (médica, HMWG).

Esta dualidade do trabalho como fonte de prazer e sofrimento encontra-se relatada na literatura, demonstrando que inexiste neutralidade na relação entre trabalho e saúde, na medida em que este favorecerá a saúde ou o adoecimento ${ }^{2}$.

Condizente com estas assertivas, segundo demonstra a Tabela 3, a maioria dos profissionais identificou, concomitantemente, aspectos favoráveis e desfavoráveis nas interfaces entre a atividade laborativa e a condição de saúde deles.

Dentre os itens positivos, a maioria destacou a remuneração - ou, mais especificamente, as condições de vida dela decorrentes - e a satisfação gerada pelo desempenho das atividades profissionais. Além desses, foram evidenciados aspectos subjetivos, a saber: possibilidade de contribuir para a melhoria do estado de saúde dos usuários, convivência com outros profissionais e reconhecimento social.

Dentre os aspectos negativos, destacou-se estresse, excesso de horas trabalhadas e ritmo intenso de atividades. Foi assinalado, ainda, que o trabalho desfavorece a saúde devido à ausência de reconhecimento e baixa remuneração, associada à necessidade de acumular atividades profissionais, o que restringe o tempo para as demais dimensões da vida. Neste aspecto, foram destacadas principalmente as condições inadequadas para a efetivação da assistência: Má condição de trabalho, má assistência por falta de materiais [...] (enfermeira, HRTM). Falta de instrumental cirúrgico adequado para dar resolutividade necessária aos casos atendidos (dentista, HMWG). Lidar com mortes evitáveis ou resultados desfavoráveis por falta de estrutura dos serviços (médica, HMWG). 
Tabela 3. Interfaces entre trabalho e saúde na perspectiva dos profissionais de hospitais públicos de urgência e emergência no Rio Grande do Norte, 2013.

\begin{tabular}{lrrrr}
\hline \multicolumn{1}{c}{ O trabalho afeta a saúde... } & HRTM & HMWG & Total \\
\hline Favoravelmente, porque a remuneração permite acesso à moradia, educação, & $\mathrm{n}$ & 47 & 81 & 128 \\
alimentação, saneamento, transporte, serviços de saúde, atividades de lazer... & $\%$ & 65,3 & 49,4 & 54,2 \\
Favoravelmente, em decorrência da satisfação que sente em realizá-lo. & $\mathrm{n}$ & 43 & 79 & 122 \\
& $\%$ & 59,7 & 48,2 & 51,7 \\
Favoravelmente, por outros motivos. & $\mathrm{n}$ & 5 & 9 & 14 \\
& $\%$ & 6,9 & 5,5 & 5,9 \\
Desfavoravelmente, devido ao estresse ocupacional. & $\mathrm{n}$ & 39 & 84 & 123 \\
& $\%$ & 54,2 & 51,2 & 52,1 \\
Desfavoravelmente, devido aos riscos de acidentes e acometimento de doenças. & $\mathrm{n}$ & 29 & 61 & 90 \\
& $\%$ & 40,3 & 37,2 & 38,1 \\
Desfavoravelmente, devido ao excesso de horas trabalhadas e/ou ao ritmo intenso & $\mathrm{n}$ & 28 & 75 & 103 \\
de atividades. & $\%$ & 38,9 & 45,7 & 43,6 \\
Desfavoravelmente, por lidar constantemente com a dor, o sofrimento e a morte. & $\mathrm{n}$ & 32 & 54 & 86 \\
& $\%$ & 44,4 & 32,9 & 36,4 \\
Desfavoravelmente, devido ao trabalho noturno e/ou durante várias horas & $\mathrm{n}$ & 30 & 55 & 85 \\
seguidas (plantões). & $\%$ & 41,7 & 33,5 & 36,0 \\
Desfavoravelmente, devido à pressão em relação aos resultados. & $\mathrm{n}$ & 17 & 42 & 59 \\
& $\%$ & 23,6 & 25,6 & 25,0 \\
Desfavoravelmente, devido à falta de tempo para descanso, lazer e & $\mathrm{n}$ & 20 & 65 & 85 \\
relacionamentos. & $\%$ & 27,8 & 39,6 & 36,0 \\
Desfavoravelmente, por outros motivos. & $\mathrm{n}$ & 13 & 22 & 35 \\
& $\%$ & 18,1 & 13,4 & 13,8 \\
\hline
\end{tabular}

São perceptíveis, portanto, as fragilidades institucionais e a gravidade de suas consequências não apenas para os profissionais, mas também para a saúde da população usuária.

É importante evidenciar que o trabalho está imbricado às demais dimensões da vida, as quais, de forma inter-relacionada, influenciam na saúde-adoecimento dos indivíduos. Nesse sentido, considerou-se relevante apreender a percepção dos sujeitos acerca das implicações do trabalho no convívio familiar e social, cujos achados estão quantitativamente demonstrados no Gráfico 1.

Conforme evidenciam majoritariamente os sujeitos $(64,6 \%)$, seu trabalho dificulta o convívio familiar/social, devido: à sobrecarga, marcada por jornadas extensas, em forma de plantões; ao trabalho noturno e em finais de semana, feriados e datas comemorativas; ao estresse e ao cansaço; à falta de tempo para o não trabalho. Estes fatores os afastam do convívio familiar e dificultam o acompanhamento mais efetivo da educação dos filhos e a participação em momentos de lazer.

Sobre os impactos do trabalho na vida familiar, estudo efetivado com profissionais de hospital universitário e seus filhos na faixa etária entre
2 e 10 anos, identificou que estas crianças apresentaram baixa qualidade de vida relacionada à saúde, principalmente nos aspectos psicossociais. Desvelou-se que a maioria tinha hábitos cotidianos não saudáveis, com excessiva exposição a computadores, videogames e televisores e ausência de atividades físicas regulares extraescolares. Subsecutivamente, as características laborais dos profissionais além de afetarem sua própria qualidade de vida e saúde, influenciam a rotina dos seus familiares ${ }^{39}$.

\section{Considerações finais}

A relação saúde-adoecimento vivenciada incessantemente pelo ser humano no percurso da vida constitui-se um fenômeno complexo e dinâmico que expressa a interação de múltiplos processos, dentre os quais o trabalho é um dos determinantes.

Os resultados permitem inferir que os profissionais reconhecem a importância do trabalho para garantia de condições materiais e subjetivas favoráveis à saúde. Entretanto, destacam predominantemente seus efeitos no desgaste físico e psí- 


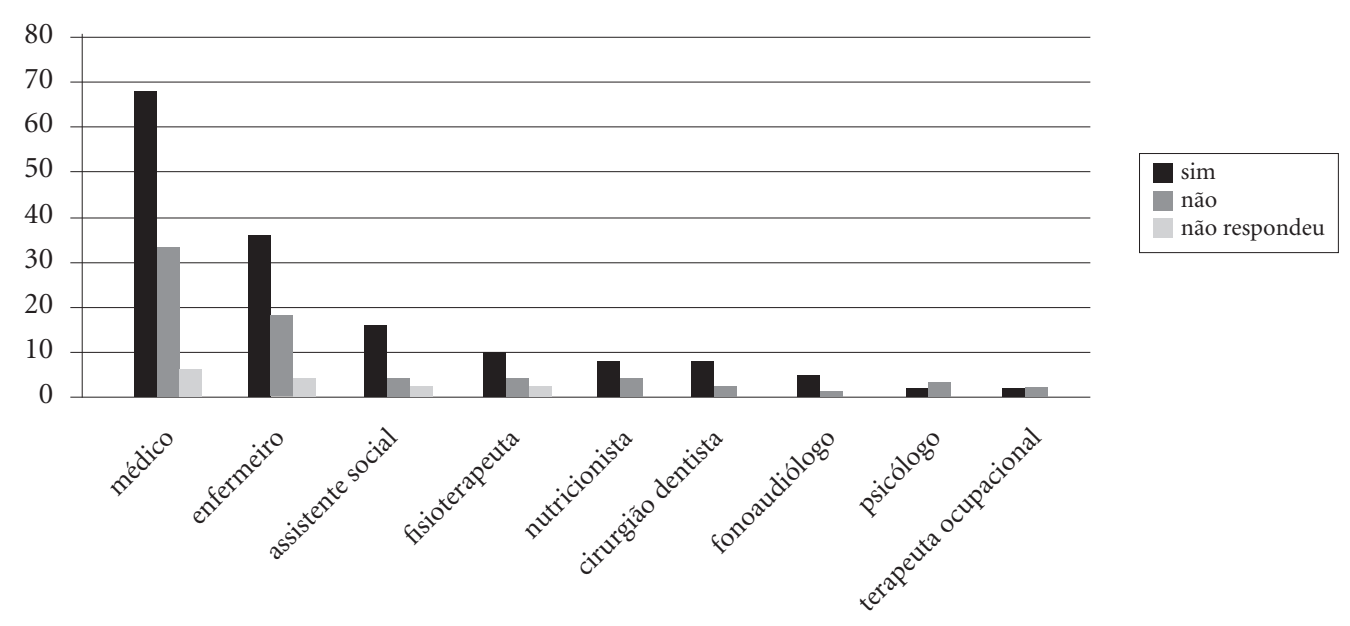

Gráfico 1. Impactos do trabalho na vida familiar/social na percepção dos profissionais de hospitais públicos de urgência e emergência do Rio Grande do Norte, 2013.

quico decorrentes de sobrecarga, turnos prolongados, expediente noturno e precárias condições de trabalho, dentre outros fatores impulsionadores de estresse, cansaço, ausência de hábitos saudáveis, hipertensão arterial, distúrbios do sono, cardiovasculares, osteomusculares e gastrintestinais.

Conclui-se, portanto, que o trabalho em saúde é permeado por contradições, pois ao passo que os trabalhadores participam da produção de saúde dos indivíduos e da coletividade, esta atividade pode ocasionar efeitos negativos na sua própria saúde, cujos impactos se espraiam na fragilização da assistência prestada à população, bem como podem interferir na qualidade de vida dos seus familiares.

Destarte, os elementos ressaltados pelos participantes deste estudo precisam ser pensados, também, como inerentes à sociabilidade capitalista e às configurações do trabalho no bojo de suas relações, não podendo ser relacionadas somente às características dos espaços de urgência e emergência, à particularidade da área da saúde ou à vinculação ao setor público, embora esses sejam elementos imprescindíveis nessa análise.

Estes problemas na gestão da política de saúde brasileira e suas interfaces com o desgaste físico e psíquico dos profissionais que trabalham neste âmbito são extremamente importantes. Com efeito, esta relação adquire maior significância pelo fato do SUS, responsável pela promoção, proteção e recuperação da saúde da população, em sua operacionalização, paradoxalmente, influenciar no desgaste da saúde dos seus trabalhadores.

Ressalva-se que o estudo particulariza a realidade de ambientes hospitalares específicos e, apesar de apresentar resultados condizentes com outros trabalhos disponíveis na literatura, não possibilita maiores generalizações. Salienta-se, ainda, que, no decurso da pesquisa, estas instituições estavam sendo alvo de reformas na sua estrutura física, o que pode ter alterado as condições de trabalho aqui relatadas.

Por fim, acredita-se que os resultados desta pesquisa podem contribuir para dar maior visibilidade ao adoecimento relacionado ao trabalho em saúde, particularmente no âmbito dos hospitais públicos de urgência e emergência, bem como para subsidiar a implementação e ampliação de ações preventivas e assistenciais direcionadas à saúde destes trabalhadores. Isto se apresenta relevante tendo em vista que a identificação e o enfrentamento dos sinais de desgaste na saúde dos participantes deste estudo podem refrear potenciais enfermidades que emergiriam tardiamente. Nesse sentido, considera-se profícua a efetivação de ações contínuas de investigação, diagnóstico e acompanhamento das condições de saúde dos profissionais no âmbito dos hospitais investigados, incluindo a avaliação de fatores de risco cardiovascular, sem descuidar de medidas direcionadas à saúde psíquica, efetivadas por profissionais especializados. 
Para tanto, é imperativo o contínuo aprofundamento do conhecimento acerca dos processos de saúde-adoecimento vivenciados pelos profissionais de saúde, o que demanda a realização de outras pesquisas, inclusive com desenho longitudinal e que abranjam indicadores clínicos e parâmetros bioquímicos na avaliação da situação de saúde destes sujeitos, no intuito de inferir relações causais entre o trabalho em serviços públicos de urgência e emergência e o adoecimento daqueles que o efetivam.

\section{Colaboradores}

IVM Rosado participou da concepção do estudo, da coleta e análise dos dados e da redação do manuscrito; GHA Russo contribuiu no planejamento da pesquisa, na análise dos dados e na redação e revisão final do artigo; EMC Maia participou da concepção do estudo, contribuiu na análise dos dados e aprovou a versão final do artigo.

\section{Agradecimentos}

Aos integrantes das instituições coparticipantes, especialmente aos profissionais que participaram da pesquisa e/ou que contribuíram para viabilizar nossa inserção nestes espaços; à professora Sâmya Ramos pelas relevantes contribuições na concepção do estudo; ao professor José Wilton Queiroz pela efetivação das análises estatísticas e à Beatriz Pereira e Suênia Cruz, pela colaboração na coleta de dados.

\section{Referências}

1. Carvalho AI, Buss PM. Determinantes sociais na saúde, na doença e na intervenção. In: Giovanella L, Escorel S, Lobato LVC, Noronha JC, Carvalho AI, organizadores. Políticas e sistemas de saúde no Brasil. Rio de Janeiro: Fiocruz; 2008. p. 141-166.

2. Pai DD, Lautert L. O trabalho em urgência e emergência e a relação com a saúde das profissionais de enfermagem. Rev. Latino-Am. Enfermagem 2008; 16(3):439444.

3. Marx K. Processo de trabalho e processo de produzir mais valia. Rio de Janeiro: Civilização Brasileira; 1980.

4. Dias EC, Oliveira RP. Machado JH, Minayo-Gomez C, Perez MAG, Hoefel MGL, Santana VS. Employment conditions and health inequities: a case study of Brazil. Cad Saude Publica 2011; 27(12):2452-2460.

5. Martins ERC, Zeitoune RCG. As condições de trabalho como fator desencadeador do uso de substancias psicoativas pelos trabalhadores de enfermagem. Esc. Anna Nery 2007; 11(4):639-644.

6. Pinheiro TMM, Dias EC, Silveira AM, Silva JM. Saúde do trabalhador. In: Campos GWS, Bonfim JRA, Minayo MCS, Akerman M, Drumond Junior M, Carvalho YM, organizadores. Tratado de saúde coletiva. $2^{\text {a }}$ ed. São Paulo: Hucitec; 2012. p. 935-964.

7. Brotto TCA, Dalbello-Araújo M. É inerente ao trabalho em saúde o adoecimento de seu trabalhador? Rev Bras Saúde Ocup 2012; 37(126):290-305.

8. O'Dwyer G, Konder MT, Machado CV, Alves CP, Ales RP. The current scenario of emergency care policies in Brasil. BMC Health Services Research 2013; 13:70.

9. Dubeux LS, Freese E, Reis YAC. Avaliação dos serviços de urgência e emergência da rede hospitalar de referência no Nordeste brasileiro. Cad Saude Publica 2010; 26(8):1508-1518.

10. Paim JS. A Constituição Cidadã e os 25 anos do Sistema Único de Saúde. Cad Saude Publica 2013; 29(10):19271953.

11. Mendes ACG, Araújo Junior AC, Furtado BMASM, Duarte PO, Silva ALA, Miranda GMD. Condições e motivações para o trabalho de enfermeiros e médicos em serviços de emergência de alta complexidade. Rev Bras Enferm 2013; 66(2):161-166.

12. Valim MD, Marziale MHP. Avaliação da exposição ocupacional a material biológico em serviços de saúde. Texto Contexto Enferm 2011; 20(Esp.):138-146. 
13. Martins MDS, Silva NAP, Correia TIG. Acidentes de trabalho e suas repercussões num hospital ao Norte de Portugal. Rev Latino-Am. Enferm 2012; 20(2):217-225.

14. Cavagioni LC, Pierin AMG. Hipertensão arterial em profissionais que atuam em serviços de atendimento pré-hospitalar. Texto Contexto Enferm 2011; 20(3):235244.

15. Governo do Estado do Rio Grande do Norte. Decreto No 22.844, de 4 de julho de 2012. Decreta estado de calamidade pública no setor hospitalar e nas unidades do serviço de saúde do Estado do Rio Grande do Norte. Diário Oficial do Estado do Rio Grande do Norte 2012; 5 jul.

16. Minayo MCS. O desafio do conhecimento: pesquisa qualitativa em saúde. $8^{\mathrm{a}}$ ed. São Paulo, Rio de Janeiro: $\mathrm{Hu}-$ citec, Abrasco; 2004.

17. Baptista DMT. O debate sobre o uso de técnicas qualitativas e quantitativas de pesquisa. In: Martinelli ML, organizadora. Pesquisa qualitativa: um instigante desafio. São Paulo: Veras; 1999. p. 31-39.

18. Bardin L. Análise de conteúdo. Lisboa: Edições 70; 2007.

19. Minayo MCS. Análise qualitativa: teoria, passos, fidedignidade. Cien Saude Colet 2012; 17(3):621-626.

20. Brasil. Ministério da Saúde (MS). Conselho Nacional de Saúde. Resolução no 196, de 10 de outubro de 1996. Diretrizes e Normas Regulamentadoras de Pesquisas Envolvendo Seres Humanos. Diário Oficial da União 1996; 16 out.

21. Brasil. Ministério da Saúde (MS). Conselho Nacional de Saúde. Resolução no 251, de 7 de agosto de 1997. Diário Oficial da União 1997; 8 ago.

22. Carrillo-García C, Solano-Ruíz MC, Martínez-Roche ME, Gómez-García CI. Influência do gênero e da idade: satisfação no trabalho de profissionais da saúde. Rev. Latino-Am. Enferm 2013; 21(6):1314-1320.

23. Feliciano KVO, Kovacs MH, Sarinho SW. burnout entre médicos da Saúde da Família: os desafios da transformação do trabalho. Cien Saude Colet 2011; 16(8):33733382.

24. Munabi IG, Buwembo W, Kitara DL, Ochieng J, Mwaka ES. Musculoskeletal disorder risk factors among nursing professionals in low resource settings: a cross-sectional study in Uganda. BMC Nursing [serial on the Internet] $2014 \mathrm{fev}$. [cited $2014 \mathrm{mar}$ 29]; 13(7). [about 8 p.]. Available from: http://www.biomedcentral.com /1472-6955/13/7.pdf

25. Lelis CM, Battaus MRB, Freitas FCT, Rocha FLR, Marziale MHP, Robazzi MLCC. Distúrbios osteomusculares relacionados ao trabalho em profissionais de enfermagem: revisão integrativa da literatura. Acta Paul Enferm 2012. 25(3):477-482.

26. Martino MMF, Abreu ACB, Barbosa MFS, Teixeira JEM. The relationship between shift work and sleep patterns in nurses. Cien Saude Colet 2013; 18(3):763768.

27. Mendes SS, Martino MMF. Trabalho em turnos: estado geral de saúde relacionado ao sono em trabalhadores de enfermagem. Rev Esc Enferm USP 2012; 46(6):14711476.
28. Esquirol Y, Perret B, Ruidavets JB, Marquie JC, Dienne E, Niezborala M, Ferrieres J. Shift work and cardiovascular risk factors: New knowledge from the past decade. Arch Cardiovasc Dis 2011; 104(12):636-668.

29. Pimenta AM, Kac G, Souza RRC, Ferreira LMBA, Siqueira SMF. Trabalho noturno e risco cardiovascular em funcionários de universidade pública. Rev Assoc Med Bras 2012; 58(2):168-177.

30. Wang F, Yeung KL, Chan WC, Kwok CC, Leung SL, Wu C, Chan EY, Yu IT, Yang XR, Tse LA. A meta-analysis on dose-response relationship between night shift work and the risk of breast cancer. Ann Oncol 2013; 24(11):2724-2732.

31. Schernhammer ES, Feskanich D, Liang G, Han J. Rotating Night-ShiftWork and Lung Cancer Risk Among Female Nurses in the United States. Am J Epidemiol 2013;178(9):1434-1441.

32. Caixeta CRCB, Borges CRC, Iwamoto HH, Camargo FC. Há "desgaste" do trabalho noturno entre os profissionais de enfermagem? Saúde Coletiva 2012; 9(57):8993.

33. Silva AA, Rotenberg L, Fischer FM. Jornadas de trabalho na enfermagem: entre necessidades individuais e condições de trabalho. Rev Saude Publica 2011; 45(6):1117-1126.

34. Neves BS, Pinheiro TMM. Perfil epidemiológico e ocupacional dos anestesiologistas inseridos no mercado de trabalho de Belo Horizonte, Minas Gerais, em 2010. Rev Bras Anestesiol 2012; 65(5):612-624.

35. Rocha MCP, Martino MMF. O estresse e qualidade de sono do enfermeiro nos diferentes turnos hospitalares. Rev Esc Enferm USP 2010; 44(2):280-286.

36. Meneghini F, Paz AA, Lautert L. Fatores ocupacionais associados aos componentes da síndrome de burnout em trabalhadores de enfermagem. Texto Contexto Enferm 2011; 20(2):225-233.

37. Vieira M, Chinelli F. Relação contemporânea entre trabalho, qualificação e reconhecimento: repercussões sobre os trabalhadores técnicos do SUS. Cien Saude Colet 2013; 18(6):1591-1600.

38. Russo GHA. Amor e dinheiro: uma relação possível? Caderno CRH 2011; 24(61):121-134.

39. Gamallo SMM, Caparroz F, Terreri MTRA, Hilário MOE, Len CA. Qualidade de vida relacionada à saúde de filhos de profissionais da área de saúde. Rev Esc Enferm USP 2012; 46(6):1313-1319.

Artigo apresentado em 03/09/2014

Aprovado em 30/04/2015

Versão final apresentada em 02/05/2015 